\title{
The Multiple Zeta Value Algebra and the Stable Derivation Algebra
}

By

Hidekazu Furusho*

\begin{abstract}
The MZV algebra is the graded algebra over $\mathbf{Q}$ generated by all multiple zeta values. The stable derivation algebra is a graded Lie algebra version of the GrothendieckTeichmüller group. We shall show that there is a canonical surjective $\mathbf{Q}$-linear map from the graded dual vector space of the stable derivation algebra over $\mathbf{Q}$ to the new-zeta space, the quotient space of the sub-vector space of the MZV algebra whose grade is greater than 2 by the square of the maximal ideal. As a corollary, we get an upper-bound for the dimension of the graded piece of the MZV algebra at each weight in terms of the corresponding dimension of the graded piece of the stable derivation algebra. If some standard conjectures by Y. Ihara and P. Deligne concerning the structure of the stable derivation algebra hold, this will become a bound conjectured in Zagier's talk at 1st European Congress of Mathematics. Via the stable derivation algebra, we can compare the new-zeta space with the $l$-adic Galois image Lie algebra which is associated with the Galois representation on the pro-l fundamental group of $\mathbf{P}_{\overline{\mathbf{Q}}}^{1}-\{0,1, \infty\}$.
\end{abstract}

\section{Contents}

$\S 0$. Introduction

$\S 1$. The MZV Algebra

$\S 1.1$. Introduction of MZV

$\S 1.2$. The MZV algebra

$\S 1.3$. The new-zeta space

Communicated by A. Tamagawa. Received August 14, 2002. Revised January 14, 2003. 2000 Mathematics Subject Classification(s): Primary 11M41.

Key words: multiple zeta values, Drinfel'd associator, stable derivation algebra.

The author is supported in part by JSPS Research Fellowships for Young Scientists.

*RIMS, Kyoto University, Kyoto 606-8502, Japan.

e-mail: furusho@kurims.kyoto-u.ac.jp 
$\S 2 . \quad$ Review of the Stable Derivation Algebra

$\S 2.1$. The stable derivation algebra

$\S 2.2$. The normalization form of the stable derivation algebra

§2.3. Weights and depths

§2.4. A connection between the $l$-adic Galois image Lie algebra and the stable derivation algebra

$\S 3$. Review of the Drinfel'd Associator

$\S 3.1$. The KZ equation and the Drinfel'd associator

§3.2. Explicit formulae

$\S 3.3$. Relations

$\S 4$. Main Results

$\S 4.1$. The new-zeta quotient of the Drinfel'd associator

\$4.2. Main theorem

$\S 4.3$. Several corollaries and conjectures

$\S 5$. Some Comparisons between 'Galois Side' and 'Hodge Side'

References

\section{$\S 0 . \quad$ Introduction}

In connection with the Galois representation on the pro-l fundamental group $\pi_{1}^{l}\left(\mathbf{P}_{\overline{\mathbf{Q}}}^{1}-\{0,1, \infty\}\right)$ for each prime $l$, the stable derivation algebra $(\mathfrak{D} .=$ $\left.\underset{w>2}{\oplus} \mathfrak{D}_{w},\left\{\mathfrak{F}^{m} \mathfrak{D} .\right\}_{m \in \mathbf{N}}\right)$ which is a certain filtered graded Lie algebra over $\mathbf{Q}$ defined combinatorially was studied independently by Y. Ihara ([14] and [16]) and V. G. Drinfel'd ([3]). It can be regarded as a graded Lie algebra version of the Grothendieck-Teichmüller group. An embedding

$$
\Psi_{l}: \mathfrak{g}^{l} \cdot \hookrightarrow \mathfrak{D} \cdot \otimes_{\mathbf{Q}} \mathbf{Q}_{l},
$$

where $\mathfrak{g}^{l}$. is the graded Lie algebra over $\mathbf{Q}_{l}$ associated to the Galois image of this pro- $l$ representation, was constructed, and it was conjectured to be an isomorphism for all primes $l$, i.e. $\mathfrak{D}$. may be a common $\mathbf{Q}$-structure of $\mathfrak{g}^{l}$. for all primes $l([12])$.

In contrast, here we shall construct a Hodge counterpart of this map. Let $Z_{w}$ be the $\mathbf{Q}$-vector space generated by all multiple zeta values of indices with weight $w$. And put $Z_{0}=\mathbf{Q}$. The $M Z V$ algebra is its formal direct sum; $Z$. $=\underset{w \geqslant 0}{\oplus} Z_{w}$. The new-zeta space is a filtered graded $\mathbf{Q}$-vector space which is a quotient of the ideal $Z_{>2} ; N Z$. $=Z_{>2} /\left(Z_{>0}\right)^{2}$. For more details, see $\S \S 1.3$. 
Theorem 4.2.2. $\quad$ There is a canonical surjective $\mathbf{Q}$-linear map

$$
\Psi_{D R}: \mathfrak{D}^{*} . \rightarrow N Z \text {. }
$$

as filtered graded $\mathbf{Q}$-vector space, where $\mathfrak{D}^{*}$. is the filtered graded dual of the stable derivation algebra.

There is a standard conjecture on the structure of $\mathfrak{D}$. which is a combination of the conjectures $(\S \S 2.4)$ by $\mathrm{Y}$. Ihara and P. Deligne concerning the Galois representation on $\pi_{1}^{l}\left(\mathbf{P}_{\overline{\mathbf{Q}}}^{1}-\{0,1, \infty\}\right)$.

Conjecture 2.4.1. $\quad \mathfrak{D}$. is a free Lie algebra generated by one element in each degree $m(=3,5,7, \ldots)$.

As a corollary of Theorem 4.2.2, we get the following

Proposition 4.3.7. If we assume Conjecture 2.4.1, then we get a "final upper bound"1

$$
\operatorname{dim}_{\mathbf{Q}} Z_{w} \leqslant d_{w}
$$

where $d_{w}$ is the conjectured value of $\operatorname{dim}_{\mathbf{Q}} Z_{w}$ in Dimension Conjecture ([23], see also $\S \S 1.2)$.

Modulo Conjecture 2.4.1, Dimension Conjecture is equivalent to

Conjecture 4.3.8. $\quad \Psi_{D R}$ is an isomorphism.

Particularly this conjecture claims that the dual vector space of $N Z$. might admit a co-Lie algebra structure deduced from that of $\mathfrak{D}^{*}$..

This paper is organized as follows. The definitions and basic known facts on multiple zeta values will be reviewed and the new zeta space will be introduced in $\S 1 . ~ \S 2$ is devoted to reviewing the stable derivation algebra which appeared in some works by Y. Ihara on the Galois representation on $\pi_{1}^{l}\left(\mathbf{P}_{\overline{\mathbf{Q}}}^{1}-\{0,1, \infty\}\right)$. In $\S 3$, we shall recall the basic properties of the Drinfel'd associator which are required to prove our main theorem. We shall state and prove our main results in $\S 4$. The connection between the new-zeta space and the stable derivation algebra will be built up there. In $\S 5$, we shall compare our story in Hodge side $(\S 4)$ with the story in Galois side (§2).

\footnotetext{
${ }^{1}$ A.B. Goncharov announced that he has shown the upper-bounding part of Dimension Conjecture by the theory of mixed Tate motives in [8]. Recently another proof was also given by $\mathrm{T}$. Terasoma in [21].
} 


\section{Acknowledgements}

I am deeply grateful to Professor Y. Ihara for introducing me to this area. This paper cannot be written without his continuous encouragements. I would also like to thank $\mathrm{H}$. Tsunogai for kindly showing me his many computations and M. Kaneko for informing me literatures on MZV's.

\section{$\S 1$. The MZV Algebra}

This section is devoted to reviewing the known facts and basic conjectures on multiple zeta values and to introducing the new-zeta space.

\section{$\S 1.1$. Introduction of MZV}

Definition 1.1.1. For each index $\mathbf{k}=\left(k_{1}, k_{2}, \ldots, k_{m}\right)$ of positive integers with $k_{1}, \ldots, k_{m-1} \geqslant 1, k_{m}>1$, the corresponding multiple zeta value (MZV for short) $\zeta(\mathbf{k})$ is, by definition, the real number defined by the convergent series:

$$
\zeta(\mathbf{k})=\sum_{\substack{0<n_{1}<\cdots<n_{m} \\ n_{i} \in \mathbf{N}}} \frac{1}{n_{1}^{k_{1}} \cdots n_{m}^{k_{m}}} .
$$

The weight of $\mathbf{k}: w t(\mathbf{k})$ (resp. the depth of $\mathbf{k}: d p(\mathbf{k})$ ) is defined as $w t(\mathbf{k})=$ $k_{1}+\cdots+k_{m}($ resp. $d p(\mathbf{k})=m)$.

Sometimes a multiple zeta value is called as a Zagier sum, a multiple harmonic series or a poly zeta value.

\section{Example 1.1.2.}

$$
\begin{aligned}
d p=1, w t=k\left(k \in \mathbf{N}_{\geqslant 2}\right) & : \zeta(k) \text { (Riemann zeta value) } \\
w t=1, & : \text { no MZV's } \\
w t=2, d p=1 & : \zeta(2) \\
w t=3, d p=1 & : \zeta(3) \\
\quad, d p=2 & : \zeta(1,2) \\
w t=4, d p=1 & : \zeta(4) \\
\quad, d p=2 & : \zeta(1,3), \zeta(2,2) \\
\quad, d p=3 & : \zeta(1,1,2) \\
w t=w(\geqslant 2), & : 2^{w-2} \text { tuples of MZV's. }
\end{aligned}
$$


Definition 1.1.3. For each natural number $w$, let $Z_{w}$ be the $\mathbf{Q}$-vector space of $\mathbf{R}$ generated by all MZV's of indices with weight $w: Z_{w}=\langle\zeta(\mathbf{k})|$ $w t(\mathbf{k})=w\rangle_{\mathbf{Q}} \subseteq \mathbf{R}$, and put $Z_{0}=\mathbf{Q}$. For each natural number $m$ and $w$, let $Z_{w}^{m}$ be the subspace of $Z_{w}$ generated by all MZV's of indices with weight $w$ and depth $m: Z_{w}^{m}=\langle\zeta(\mathbf{k}) \mid w t(\mathbf{k})=w, d p(\mathbf{k})=m\rangle_{\mathbf{Q}}$.

$Z_{w}$ has the ascending depth filtration: $Z_{w}^{\leqslant m}=\langle\zeta(\mathbf{k})| w t(\mathbf{k})=w, d p(\mathbf{k}) \leqslant$ $m\rangle_{\mathbf{Q}}$.

$$
\langle\zeta(w)\rangle_{\mathbf{Q}}=Z_{w}^{\leqslant 1} \subseteq Z_{w}^{\leqslant 2} \subseteq \cdots \subseteq Z_{w}^{\leqslant w-1}=Z_{w} \subset \mathbf{R}
$$

On $Z_{0}$, set $Z_{0}=Z_{0}^{\leqslant 0}=Z_{0}^{\leqslant 1}=Z_{0}^{\leqslant 2}=\cdots$.

We should note that $Z_{w}$ is not graded by depths. For example, see $\zeta(3)=$ $\zeta(1,2), \zeta(1,3)=\frac{1}{4} \zeta(4)$.

\section{$\S 1.2$. The MZV algebra}

Let $Z$. be the formal direct sum of $Z_{w}$ for all $w \geqslant 0 ; Z$. $=\underset{w \geqslant 0}{\oplus} Z_{w}$. Put $Z_{>0}=\underset{w>0}{\oplus} Z_{w}$. They are $\mathbf{Q}$-vector spaces graded by weights.

Lemma 1.2.1. $\quad$ The product of two $M Z V$ 's, one with weight a and depth $m$, the other with weight $b$ and depth $l$ belongs to the vector space $Z_{a+b}^{\leqslant l+m}$.

This follows directly from the definitions. Considering $Z$. as the sub-vector space of the graded algebra $S .=\underset{w \geqslant 0}{\oplus} S_{w}$ where $S_{w}=\mathbf{R}$, we can give $Z$. a structure of algebra.

Property 1.2.2. $\quad Z$. becomes a filtered graded Q-algebra (i.e. $Z_{a}^{\leqslant l}$. $Z_{b}^{\leqslant m} \subseteq Z_{a+b}^{\leqslant l+m}$ ) and $Z_{>0}$ is a homogeneous ideal of $Z$..

We shall call $Z$. the MZV algebra. There is a natural ring homomorphism $d: Z$. $\rightarrow \mathbf{R}$ which is identity on $Z_{w}$ for each $w$. The following conjecture is stated in [5] and [8].

Direct Sum Conjecture. The homomorphism $d$ is injective, i.e. there are no non-trivial $\mathbf{Q}$-linear relations among different weight MZV's.

This conjecture would imply that all MZV's are transcendental numbers. But not much is known about these transcendencies. Among the known results, we quote here the following; $\zeta(2 k)(k=1,2, \ldots)$ is a rational multiple of $\pi^{2 k}$ (L. Euler), it is transcendental (Lindemann), $\zeta(3)$ is irrational (R. Apéry) and $\{\zeta(n) \notin \mathbf{Q} \mid n=3,5,7, \ldots\}$ is an infinite set, which is a recent result of Tanguy Rivoal [20]. It seems hard to prove the direct sum conjecture. On the dimension of $Z$., the following conjecture appears in [23]. 
Dimension Conjecture. $\operatorname{dim}_{\mathbf{Q}} Z_{w}$ is equal to $d_{w}$, which is given by the Fibonacci-like recurrence $d_{w}=d_{w-2}+d_{w-3}$, with initial values $d_{0}=1, d_{1}=$ $0, d_{2}=1$, i.e.

$$
d_{w}=-\frac{\alpha^{w+2}(\beta-\gamma)+\beta^{w+2}(\gamma-\alpha)+\gamma^{w+2}(\alpha-\beta)}{(\alpha-\beta)(\beta-\gamma)(\gamma-\alpha)},
$$

where $\alpha, \beta, \gamma$ are roots of $x^{3}-x-1$ among which $\alpha$ is the only real root $\alpha=1.324717 \cdots$, and $|\beta|=|\gamma|<1$.

By the above formula for $d_{w}$, approximately $d_{w} \sim \frac{\alpha^{w+2}}{(\alpha-\beta)(\alpha-\gamma)}=$ $(0.411496 \cdots) \cdot(1.324717 \cdots)^{w}$, so $d_{w}$ is much smaller than $2^{w-2}$, the number of indices of $w t=w$. So it indicates that there must be many Q-linear relations among MZV's of the same weight. In fact, several such relations have been found by many mathematicians and physicists. For example, $\zeta(3)=\zeta(1,2)$ and $\zeta(4)=4 \zeta(1,3)=\frac{4}{3} \zeta(2,2)$ by L. Euler. Due to those relations we know generators of the MZV algebra in lower weights in Example 1.2.3 and the upper bound $\operatorname{dim}_{\mathbf{Q}} Z_{w} \leqslant d_{w}$ for $w \leqslant 12$ (for $d_{w}$, see also below). But it seems difficult to prove the lower-bounding inequalities because we need to show the linear independency.

\begin{tabular}{|c||c|c|c|c|c|c|c|c|c|c|c|c|c|}
\hline$w$ & 0 & 1 & 2 & 3 & 4 & 5 & 6 & 7 & 8 & 9 & 10 & 11 & 12 \\
\hline$d_{w}$ & 1 & 0 & 1 & 1 & 1 & 2 & 2 & 3 & 4 & 5 & 7 & 9 & 12 \\
\hline $2^{w-2}$ & $/$ & $/$ & 1 & 2 & 4 & 8 & 16 & 32 & 64 & 128 & 256 & 512 & 1024 \\
\hline
\end{tabular}

\section{Example 1.2.3.}

$$
\begin{aligned}
Z_{0} & =\langle 1\rangle_{\mathbf{Q}} \\
Z_{1} & =0 \\
Z_{2} & =\left\langle\pi^{2}\right\rangle_{\mathbf{Q}} \\
Z_{3} & =\langle\underline{\zeta(3)}\rangle_{\mathbf{Q}} \\
Z_{4} & =\left\langle\pi^{4}\right\rangle_{\mathbf{Q}} \\
Z_{5} & =\left\langle\pi^{2} \zeta(3), \underline{\zeta(5)}\right\rangle_{\mathbf{Q}} \\
Z_{6} & =\left\langle\pi^{6}, \zeta(3)^{2}\right\rangle_{\mathbf{Q}} \\
Z_{7} & =\left\langle\pi^{4} \zeta(3), \pi^{2} \zeta(5), \underline{\zeta(7)}\right\rangle_{\mathbf{Q}} \\
Z_{8} & =\left\langle\pi^{8}, \pi^{2} \zeta(3)^{2}, \zeta(3) \zeta(5), \underline{\zeta(3,5)\rangle_{\mathbf{Q}}}\right. \\
Z_{9} & =\left\langle\pi^{6} \zeta(3), \pi^{4} \zeta(5), \pi^{2} \zeta(7), \zeta(3)^{3}, \zeta(9)\right\rangle_{\mathbf{Q}} \\
Z_{10} & =\left\langle\pi^{10}, \pi^{4} \zeta(3)^{2}, \pi^{2} \zeta(3) \zeta(5), \pi^{2} \zeta(3,5), \zeta(3) \zeta(7), \zeta(5)^{2}, \underline{\zeta(3,7)}\right\rangle_{\mathbf{Q}}
\end{aligned}
$$

For weights 11 and 12, see Example 4.3.4. 


\section{$\S 1.3 . \quad$ The new-zeta space}

It seems now that there exist no non-trivial Q-linear relations among the above MZV's at each weight in Examples 1.2.3. So if the dimension conjecture is true, they must form bases of the vector spaces in respective weights. Compare the underlined MZV's with the other ones. The other ones are old-comers in the sense that they are written as a product of lower weight MZV's. In contrast, the underlined ones might be new-comers. Those new-zeta values are algebraic generators of the MZV algebra $Z$.. Where and how many those new-zeta values appear? On this question, it is natural to take the following quotient space of $Z$.

Definition 1.3.1. The new-zeta space is the graded vector space over $\mathbf{Q}$.

$$
N Z \cdot=\underset{w \geqslant 1}{\oplus} N Z_{w}:=Z \cdot /\left(\left(Z_{>0}\right)^{2} \oplus \mathbf{Q} \cdot \pi^{2} \oplus \mathbf{Q} \cdot 1\right)=Z_{>2} /\left(Z_{>0}\right)^{2},
$$

where $\left(Z_{>0}\right)^{2}$ is the ideal of $Z$. generated by products of the two elements in $Z_{>0}$. It is equipped with the depth filtration $\left\{N Z:{ }^{\leqslant}\right\}_{m \in \mathbf{N}}$ which is induced from the depth filtration $\{Z \leqslant m\}_{m \in \mathbf{N}}$ of $Z$. by the natural surjection $Z$. $\rightarrow N Z$.

It is also natural to raise the following

Problem 1.3.2. Clarify the structure of $\left(N Z .,\{N Z . \leqslant m\}_{m \in \mathbf{N}}\right)$ as the filtered graded $\mathbf{Q}$-vector space.

This problem is one of our main motivations in this paper. We note that the algebra structure of $N Z$. is no more interesting, because its multiplicative map is trivial.

\section{§2. Review of the Stable Derivation Algebra}

In this section, we shall recall the definitions on the stable derivation algebra which appeared in works by Ihara on Galois representation on $\pi_{1}\left(\mathbf{P}_{\overline{\mathbf{Q}}}^{1}\right.$ $\{0,1, \infty\})([11] \sim[16])$.

\section{§2.1. The stable derivation algebra}

Let $n \geqslant 4$. The braid Lie algebra on $n$ strings $\mathfrak{P}^{(n)}=\underset{k \geqslant 1}{\oplus} \mathfrak{P}_{k}^{(n)}$ is the graded Lie algebra over $\mathbf{Q}$ which has the following presentation.

Generators $\quad x_{i, j} \quad(1 \leqslant i, j \leqslant n)$ 
Relations (a) $x_{i, i}=0, x_{i, j}=x_{j, i} \quad(1 \leqslant i, j \leqslant n)$
(b) $\sum_{k=1}^{5} x_{i, k}=0 \quad(1 \leqslant i \leqslant n)$
(c) $\left[x_{i, j}, x_{k, l}\right]=0 \quad(\{i, j\} \cap\{k, l\}=\emptyset)$

Grading $\quad \operatorname{deg} x_{i, j}=1 \quad(1 \leqslant i<j \leqslant n)$

It is obtained from the lower central series of the pure braid group on $n$ strings in a certain standard manner (see [13]). Note that $\mathfrak{P}^{(4)}$ is freely generated by $x_{1,2}$ and $x_{2,3}$, and $\mathfrak{P}^{(5)}$ is an extension of the free Lie algebra of rank 2 by that of rank 3. As for general $\mathfrak{P}^{(n)}$, they are connected with each other by the standard projections $p_{n}: \mathfrak{P}^{(n+1)} \rightarrow \mathfrak{P}^{(n)}$ defined by $x_{i, j} \mapsto x_{i, j}(1 \leqslant i, j \leqslant n)$ and $x_{i, j} \mapsto 0$ (otherwise).

The derivation $D$ of $\mathfrak{P}^{(n)}$ is called special if there exists some $t_{i, j} \in \mathfrak{P}^{(n)}$ such that $D\left(x_{i, j}\right)=\left[t_{i, j}, x_{i, j}\right]$ for each $i, j(1 \leqslant i, j \leqslant n)$. The special derivations of $\mathfrak{P}^{(n)}$ form the graded Lie algebra $\operatorname{Der}^{\sharp} \mathfrak{P}^{(n)}$ whose degree $k(k \geqslant 1)$ part consists of those $D$ 's with $t_{i, j} \in \mathfrak{P}_{k}^{(n)}(1 \leqslant i, j \leqslant n)$. This Lie algebra contains the inner derivations InnDer $\mathfrak{P}^{(n)}$ as homogeneous ideal and the quotient Lie algebra OutDer $\mathfrak{P}^{(n)}:=\operatorname{Der}^{\sharp} \mathfrak{P}^{(n)} / \operatorname{InnDer} \sharp \mathfrak{P}^{(n)}$ is called the graded Lie algebra of special outer derivations of $\mathfrak{P}^{(n)}$. The symmetric group $\mathfrak{S}_{n}$ acts on $\mathfrak{P}^{(n)}$ as automorphisms of graded Lie algebra by $x_{i, j} \mapsto x_{\sigma(i), \sigma(j)}\left(\sigma \in \mathfrak{S}_{n}\right)$ for each $i, j(1 \leqslant i, j \leqslant n)$. The $\mathfrak{S}_{n}$-action $D \mapsto \sigma \circ D \circ \sigma^{-1}$ for each derivation $D$ of $\mathfrak{P}^{(n)}\left(\sigma \in \mathfrak{S}_{n}\right)$ induces an $\mathfrak{S}_{n}$-action on $\operatorname{OutDer}^{\sharp} \mathfrak{P}^{(n)}$. The invariant subalgebra $\mathfrak{D}^{(n)}:=\left(\text { OutDer } \mathfrak{P}^{(n)}\right)^{\mathfrak{S}_{n}}$ of this action is called the graded Lie algebra of symmetric special outer derivations of $\mathfrak{P}^{(n)}$. Note that each degree $k$ piece $\mathfrak{D}_{k}^{(n)}$ of $\mathfrak{D}^{(n)}=\underset{k \geqslant 1}{\oplus} \mathfrak{D}_{k}^{(n)}$ is finite dimensional $\mathbf{Q}$-vector space.

Since each special derivation $D$ of $\mathfrak{P}^{(n+1)}$ leaves $\operatorname{Kerp}_{n}$ stable, we can associate the Lie algebra homomorphism $\psi_{n}: \mathfrak{D}^{(n+1)} \rightarrow \mathfrak{D}^{(n)}$. On the following sequence of Lie algebra homomorphisms

$$
\cdots \stackrel{\psi_{n+1}}{\rightarrow} \mathfrak{D}^{(n+1)} \stackrel{\psi_{n}}{\rightarrow} \mathfrak{D}^{(n)} \stackrel{\psi_{n-1}}{\rightarrow} \cdots \cdots \stackrel{\psi_{5}}{\rightarrow} \mathfrak{D}^{(5)} \stackrel{\psi_{4}}{\rightarrow} \mathfrak{D}^{(4)},
$$

Y. Ihara proved that $\psi_{n}$ is injective for $n \geqslant 4$ in [13], and then in [14], that $\psi_{n}$ is bijective for $n \geqslant 5$. Therefore, $\psi_{n}$ induces

$$
\cdots \simeq \mathfrak{D}^{(n+1)} \simeq \mathfrak{D}^{(n)} \simeq \cdots \cdots \simeq \mathfrak{D}^{(5)} \hookrightarrow \mathfrak{D}^{(4)} .
$$

After the stability property of this tower for $n \geqslant 5$, he called $\mathfrak{D}^{(5)}$ the stable derivation algebra. The sense of considering such a tower of Lie algebras is 
explained in [12] in connection with the action of the absolute Galois group $\operatorname{Gal}(\overline{\mathbf{Q}} / \mathbf{Q})$ on the pro- $l$ fundamental group of $\mathbf{P}_{\overline{\mathbf{Q}}}^{1}-\{0,1, \infty\}$ and partly it concerns the philosophy of "Teichmüller-Lego" in [9].

\section{§2.2. The normalization form of the stable derivation algebra}

Let $\mathbb{L} .=\underset{w \geqslant 1}{\oplus} \mathbb{L}_{w}=\mathbf{Q} x \oplus \mathbf{Q} y \oplus \mathbf{Q}[x, y] \oplus \mathbf{Q}[x,[x, y]] \oplus \mathbf{Q}[y,[x, y]] \oplus \cdots$ be the free graded Lie algebra over $\mathbf{Q}$ on two variables $x$ and $y$ with grading $\operatorname{deg} x=$ $\operatorname{deg} y=1$. This Lie algebra $\mathbb{L}$. has the descending filtration $\left\{\mathfrak{F}^{m} \mathbb{L} .\right\}_{m \in \mathbf{N}}$ : $\mathbb{L} .=\mathfrak{F}^{1} \mathbb{L} . \supseteq \mathfrak{F}^{2} \mathbb{L} . \supseteq \mathfrak{F}^{3} \mathbb{L} . \supseteq \mathfrak{F}^{4} \mathbb{L} . \supseteq \cdots \cdots$, which is defined inductively by $\mathfrak{F}^{1} \mathbb{L} .=\mathbf{Q} y \oplus \mathbf{Q}[x, y] \oplus \mathbf{Q}[x,[x, y]] \oplus \mathbf{Q}[y,[x, y]] \oplus \cdots$ and $\mathfrak{F}^{m} \mathbb{L} .=\left[\mathfrak{F}^{1} \mathbb{L} ., \mathfrak{F}^{m-1} \mathbb{L}.\right]$.

It was shown in [14] that the stable derivation algebra $\mathfrak{D}^{(5)}$ can be identified with the graded Lie subalgebra $\mathfrak{D}$. of Der $\mathbb{L}$. which has the following presentation: $\mathfrak{D} \cdot=\underset{w \geqslant 1}{\oplus} \mathfrak{D}_{w}$,

where $\mathfrak{D}_{w}=\left\{D_{f} \in \operatorname{Der} \mathbb{L} \cdot \mid f \in \mathbb{L}_{w}\right.$ satisfies (0) (iii) below. $\}$

$$
\left\{\begin{array}{l}
\text { (0) } f \in[\mathbb{L} ., \mathbb{L} .] \quad\left(=\underset{k=2}{\infty} \mathbb{L}_{k}\right) \\
\text { (i) } f(x, y)+f(y, x)=0 \\
\text { (ii) } f(x, y)+f(y, z)+f(z, x)=0 \text { for } x+y+z=0 \\
\text { (iii) } \sum_{i \in \mathbb{Z} / 5} f\left(x_{i, i+1}, x_{i+1, i+2}\right)=0 \text { in } \mathfrak{P}^{(5)} .
\end{array}\right.
$$

Here, for any Lie algebra $H$ and $\alpha, \beta \in H, f(\alpha, \beta)$ denotes the image of $f \in \mathbb{L}$. by the homomorphism $\mathbb{L}$. $\rightarrow H$ defined by $x \mapsto \alpha, y \mapsto \beta$ and, for $f$ in $\mathbb{L}$. $D_{f}: \mathbb{L} . \rightarrow \mathbb{L}$. is a special derivation defined by $D_{f}(x)=0$ and $D_{f}(y)=[y, f]$. It can be checked easily that $\left[D_{f}, D_{g}\right]=D_{h}$ with $h=[f, g]+D_{f}(g)-D_{g}(f)$. Note that $D \in \mathfrak{D}$. determines uniquely $f \in[\mathbb{L}$., $\mathbb{L}$.$] such that D=D_{f}$. We also remark that in fact (iii) implies (i).

The Lie algebra $\mathfrak{D}$. was also studied independently by V. G. Drinfel'd ([3]) as the graded $\mathbf{Q}$-Lie algebra version of the Grothendieck-Teichmüller group. In [3], $\mathfrak{D}$. and $\mathfrak{P}^{(5)}$ appeared as $\oplus \mathfrak{g r t} \mathfrak{t}_{1}^{n}(\mathbb{Q})$ and $\mathfrak{a}_{4}^{\mathbb{Q}}$.

Remark 2.2.1. In [14] and [16], (ii) is replaced by

$$
[y, f(x, y)]+[z, f(x, z)]=0 \quad \text { for } x+y+z=0 .
$$

But (i), (ii), (iii) and (i), (ii)', (iii) are equivalent (cf. [3] and [14]).

From now on, we will identify the stable derivation algebra $\mathfrak{D}^{(5)}$ with $\mathfrak{D}$. 


\section{$\S 2.3$. Weights and depths}

Against the standard terminologies in graded Lie algebras, degrees of the stable derivation algebra are called weights in connection with weights of modular forms in [16]. The depth filtration of $\mathfrak{D}$. which is introduced in [16] is the descending filtration $\left\{\mathfrak{F}^{m} \mathfrak{D} .\right\}_{m \in \mathbf{N}}$ :

$$
\text { D. }=\mathfrak{F}^{1} \mathfrak{D} . \supseteq \mathfrak{F}^{2} \mathfrak{D} . \supseteq \mathfrak{F}^{3} \mathfrak{D} . \supseteq \cdots
$$

where $\quad \mathfrak{F}^{m} \mathfrak{D} .=\left\{D_{f} \mid f \in \mathfrak{F}^{m} \mathbb{L}\right.$. satisfies $(0) \sim($ iii $\left.)\right\}$.

It provides $\mathfrak{D}$. with a structure of filtered graded Lie algebra. In $\S 4$, we shall see that these terminologies, weight and depth, correspond to those of MZV's. On the dimension of the depth filtration of the stable derivation algebra at each weight $w$, the following results are known.

Proposition 2.3.1 [16].

- $\mathfrak{F}^{m} \mathfrak{D}_{w}=\mathfrak{F}^{m+1} \mathfrak{D}_{w}$ if $w \not \equiv m(\bmod 2)$.

- $\mathfrak{F}^{m} \mathfrak{D}_{w}=\{0\}$ if $m>\frac{w}{2}$.

- $\operatorname{dim}_{\mathbf{Q}}\left(\mathfrak{F}^{1} \mathfrak{D}_{w} / \mathfrak{F}^{2} \mathfrak{D}_{w}\right)= \begin{cases}1 & w=3,5,7,9, \ldots \\ 0 & w: \text { otherwise }\end{cases}$

- $\operatorname{dim}_{\mathbf{Q}}\left(\mathfrak{F}^{2} \mathfrak{D}_{w} / \mathfrak{F}^{3} \mathfrak{D}_{w}\right)=\left\{\begin{array}{cl}0 & w: \text { odd } \\ {\left[\frac{w-2}{6}\right]} & w: \text { even }\end{array}\right.$ (Ihara-Takao).

Example 2.3.2. The following computation table of the stable derivation algebra up to weight 12 is due to Y. Ihara, M. Matsumoto and H. Tsunogai.

$$
\begin{aligned}
& \mathfrak{D}_{1}=0, \\
& \mathfrak{D}_{2}=0, \\
& \mathfrak{D}_{3}=\left\langle D_{f_{3}}\right\rangle_{\mathbf{Q}}, \\
& \mathfrak{D}_{4}=0, \\
& \mathfrak{D}_{5}=\left\langle D_{f_{5}}\right\rangle_{\mathbf{Q}}, \\
& \mathfrak{D}_{6}=0, \\
& \mathfrak{D}_{7}=\left\langle D_{f_{7}}\right\rangle_{\mathbf{Q}}, \\
& \mathfrak{D}_{8}=\left\langle\left[D_{f_{3}}, D_{f_{5}}\right]\right\rangle_{\mathbf{Q}}, \\
& \mathfrak{D}_{9}=\left\langle D_{f_{9}}\right\rangle_{\mathbf{Q}}, \\
& \mathfrak{D}_{10}=\left\langle\left[D_{f_{3}}, D_{f_{7}}\right]\right\rangle_{\mathbf{Q}}, \\
& \mathfrak{D}_{11}=\left\langle D_{f_{11}},\left[D_{f_{3}},\left[D_{f_{3}}, D_{f_{5}}\right]\right]\right\rangle_{\mathbf{Q}}, \\
& \mathfrak{D}_{12}=\left\langle\left[D_{f_{3}}, D_{f_{9}}\right],\left[D_{f_{5}}, D_{f_{7}}\right]\right\rangle_{\mathbf{Q}} .
\end{aligned}
$$


Here, $f_{w}$ is a certain element of $\mathbb{L}_{w}$. For example, $f_{3}$ and $f_{5}$ are as given below.

$$
\begin{aligned}
f_{3}= & {[x,[x, y]]+[y,[x, y]] } \\
f_{5}= & 2[x,[x,[x,[x, y]]]]+4[y,[x,[x,[x, y]]]]+4[y,[y,[x,[x, y]]]] \\
& +2[y,[y,[y,[x, y]]]]+[[x, y],[x,[x, y]]]+3[[x, y],[y,[x, y]]] .
\end{aligned}
$$

\begin{tabular}{|c||c|c|c|c|c|c|c|c|c|c|c|c|}
\hline$w$ & 1 & 2 & 3 & 4 & 5 & 6 & 7 & 8 & 9 & 10 & 11 & 12 \\
\hline $\operatorname{dim}_{\mathbf{Q}} \mathfrak{D}_{w}\left(=\mathfrak{F}^{1} \mathfrak{D}_{w}\right)$ & 0 & 0 & 1 & 0 & 1 & 0 & 1 & 1 & 1 & 1 & 2 & 2 \\
\hline \hline $\operatorname{dim}_{\mathbf{Q}} \mathfrak{F}^{2} \mathfrak{D}_{w}$ & 0 & 0 & 0 & 0 & 0 & 0 & 0 & 1 & 0 & 1 & 1 & 2 \\
\hline $\operatorname{dim}_{\mathbf{Q}} \mathfrak{F}^{3} \mathfrak{D}_{w}$ & 0 & 0 & 0 & 0 & 0 & 0 & 0 & 0 & 0 & 0 & 1 & 1 \\
\hline $\operatorname{dim}_{\mathbf{Q}} \mathfrak{F}^{4} \mathfrak{D}_{w}$ & 0 & 0 & 0 & 0 & 0 & 0 & 0 & 0 & 0 & 0 & 0 & 1 \\
\hline $\operatorname{dim}_{\mathbf{Q}} \mathfrak{F}^{5} \mathfrak{D}_{w}$ & 0 & 0 & 0 & 0 & 0 & 0 & 0 & 0 & 0 & 0 & 0 & 0 \\
\hline
\end{tabular}

A remarkable phenomena are pointed out in [11] (see also [16]).

$$
2\left[D_{f_{3}}, D_{f_{9}}\right]-27\left[D_{f_{5}}, D_{f_{7}}\right] \in \mathfrak{F}^{3} \mathfrak{D} .
$$

\section{§2.4. A connection between the $l$-adic Galois image Lie algebra and the stable derivation algebra}

Let $l$ be a prime. Consider the outer action

$$
\varphi_{l}: \operatorname{Gal}(\overline{\mathbf{Q}} / \mathbf{Q}) \rightarrow \text { Out } \pi_{1}^{(l)}\left(\mathbf{P}_{\overline{\mathbf{Q}}}^{1}-\{0,1, \infty\}\right)
$$

of the absolute Galois group $\operatorname{Gal}(\overline{\mathbf{Q}} / \mathbf{Q})$ on the pro- $l$ fundamental group of $\mathbf{P}_{\overline{\mathbf{Q}}}^{1}-\{0,1, \infty\}$. The l-adic Galois image Lie algebra $\mathfrak{g}^{l}=\underset{w \geqslant 1}{\oplus} \mathfrak{g}_{w}^{l}$ is the graded Lie algebra over $\mathbf{Q}_{l}$ which is constructed from the image of $\varphi_{l}$ in a certain standard way and it is shown that $\mathfrak{g}^{l}$. can be embedded into the $l$-adic stable derivation algebra

$$
\Psi_{l}: \mathfrak{g}^{l} \hookrightarrow \mathfrak{D} \otimes_{\mathbf{Q}} \mathbf{Q}_{l}
$$

for all primes $l$ (for more details, see [12]). On the map $\Psi_{l}$ and these Lie algebras, $\mathfrak{g}^{l}$. and $\mathfrak{D}$., the following conjectures are stated in [12] and [16] and partly in $[2]$.

\section{Conjecture 2.4.1.}

(1) $\Psi_{l}: \mathfrak{g}^{l} \hookrightarrow \hookrightarrow \mathfrak{D} . \otimes_{\mathbf{Q}} \mathbf{Q}_{l}$ is an isomorphism for every prime $l$.

(2) These Lie algebras are free.

(3) On generators of these Lie algebras, we can take one element in each odd degree $m(m=3,5,7, \ldots)$. 
The validity of (1) implies that the $l$-adic Galois image Lie algebra $\mathfrak{g}^{l} \cdot$ has a common $\mathbf{Q}$-structure for all primes $l$ and the stable derivation algebra $\mathfrak{D}$. which is defined entirely combinatorially becomes this common Q-structure. Conjecture (3) for $\mathfrak{g}^{l}$. was proved by R. Hain and M. Matsumoto in [10]. On the lower weight up to 12 for $\mathfrak{D}$. and 17 for $\mathfrak{g}^{l}$., H. Tsunogai verified (1), (2) and (3) by computations ([22]).

\begin{tabular}{|c||c|c|c|c|c|c|c|c|c|c|c|c|c|c|c|c|c|}
\hline$w$ & 1 & 2 & 3 & 4 & 5 & 6 & 7 & 8 & 9 & 10 & 11 & 12 & 13 & 14 & 15 & 16 & 17 \\
\hline $\operatorname{dim}_{\mathbf{Q}} \mathfrak{D}_{w}$ & 0 & 0 & 1 & 0 & 1 & 0 & 1 & 1 & 1 & 1 & 2 & 2 & $/$ & $/$ & $/$ & $/$ & $/$ \\
\hline $\operatorname{dim}_{\mathbf{Q}} \mathfrak{g}_{w}^{l}$ & 0 & 0 & 1 & 0 & 1 & 0 & 1 & 1 & 1 & 1 & 2 & 2 & 3 & 3 & 4 & 4 or 5 & 7 \\
\hline
\end{tabular}

\section{$\S 3 . \quad$ Review of the Drinfel'd Associator}

We shall review the basic properties of the Drinfel'd associator which is required to prove our main theorem in the next section. Most of them can be found in [3] and [17].

\section{§3.1. The KZ equation and the Drinfel'd associator}

Let $A_{\mathbb{C}}^{\wedge}=\mathbb{C}\langle\langle A, B\rangle\rangle$ be the non-commutative formal power series ring with complex number coefficients generated by two elements $A$ and $B$. Consider the Knizhnik-Zamolodchikov equation (KZ equation for short)

$$
\frac{\partial G}{\partial u}(u)=\left(\frac{A}{u}+\frac{B}{u-1}\right) \cdot G(u)
$$

where $G(u)$ is an analytic function in complex variable $u$ with values in $A_{\mathbb{C}}^{\wedge}$ where 'analytic' means each of whose coefficient is analytic. The equation (KZ) has singularities only at 0,1 and $\infty$. Let $\mathbb{C}^{\prime}$ be the complement of the union of the real half-lines $(-\infty, 0]$ and $[1,+\infty)$ in the complex plane. This is a simply-connected domain. The equation (KZ) has a unique analytic solution on $\mathbb{C}^{\prime}$ having a specified value at any given points on $\mathbb{C}^{\prime}$. Moreover, at the singular points 0 and 1 , there exist unique solutions $G_{0}(u)$ and $G_{1}(u)$ of $(\mathrm{KZ})$ such that

$$
G_{0}(u) \approx u^{A}(u \rightarrow 0), \quad G_{1}(u) \approx(1-u)^{B}(u \rightarrow 1),
$$

where $\approx$ means that $G_{0}(u) \cdot u^{-A}$ (resp. $G_{1}(u) \cdot(1-u)^{-B}$ ) has an analytic continuation in a neighborhood of 0 (resp. 1) with value 1 at 0 (resp. 1). Here, $u^{A}:=\exp (A \log u):=1+\frac{A \log u}{1 !}+\frac{(A \log u)^{2}}{2 !}+\frac{(A \log u)^{3}}{3 !}+\cdots$ and $\log u:=\int_{1}^{u} \frac{d t}{t}$ 
in $\mathbb{C}^{\prime}$. In the same way, $(1-u)^{B}$ is well-defined on $\mathbb{C}^{\prime}$. One can calculate the lower degree parts of $G_{0}(u)$ as follows.

$$
\begin{aligned}
G_{0}(z) & =1+(\log z) A+\log (1-z) B+\frac{(\log z)^{2}}{2} A^{2}-L i_{2}(z) A B \\
& +\left\{L i_{2}(z)+(\log z) \log (1-z)\right\} B A+\frac{\{\log (1-z)\}^{2}}{2} B^{2}+\frac{(\log z)^{3}}{6} A^{3} \\
& -L i_{3}(z) A^{2} B+\left\{2 L i_{3}(z)+(\log z) L i_{2}(z)\right\} A B A+L i_{1,2}(z) A B^{2} \\
& -\left[L i_{3}(z)-(\log z) L i_{2}(z)-\frac{(\log z)^{2} \log (1-z)}{2}\right] B A^{2}+L i_{2,1}(z) B A B \\
& -\left[L i_{1,2}(z)+L i_{2,1}(z)-\frac{\log z\{\log (1-z)\}^{2}}{2}\right] B^{2} A \\
& +\frac{\{\log (1-z)\}^{3}}{6} B^{3}+\cdots
\end{aligned}
$$

where

$$
L i_{k_{1}, \ldots, k_{m}}(z):=\sum_{\substack{0<n_{1}<\cdots<n_{m} \\ n_{i} \in \mathbf{N}}} \frac{z^{n_{m}}}{n_{1}^{k_{1}} \cdots n_{m}^{k_{m}}}
$$

Since $G_{0}(u)$ and $G_{1}(u)$ are both non-zero unique solutions of (KZ) with the specified asymptotic behaviors, they must coincide with each other up to multiplication by an invertible element of $A_{\overparen{C}}^{\wedge}$.

Definition 3.1.1. The Drinfel'd associator ${ }^{2}$ is the element $\Phi_{K Z}(A, B)$ of $A_{\mathbb{C}}^{\wedge}$ which is defined by

$$
G_{0}(u)=G_{1}(u) \cdot \Phi_{K Z}(A, B) .
$$

By considering the image in $\left(A_{\mathbb{C}}^{\wedge}\right)^{a b}$, the abelianization of $A_{\mathbb{C}}^{\wedge}$, we easily find that $\Phi_{K Z}(A, B) \equiv 1$ in $\left(A_{\mathbb{C}}^{\wedge}\right)^{a b}$.

\section{§3.2. Explicit formulae}

We will discuss on each coefficient of the Drinfel'd associator $\Phi_{K Z}(A, B)$.

Let $\omega_{1}, \omega_{2}, \ldots, \omega_{n}(n \geqslant 1)$ be differential 1 -forms on $\mathbb{C}^{\prime}$. An iterated integral $\int_{0}^{1} \omega_{n} \circ \omega_{n-1} \circ \cdots \circ \omega_{1}$ is defined inductively as $\int_{0}^{1} \omega_{n}\left(t_{n}\right) \int_{0}^{t_{n}} \omega_{n-1} \circ \cdots \circ \omega_{1}$.

\footnotetext{
${ }^{2}$ To be precise, Drinfel'd defined $\varphi_{K Z}(A, B)$ instead of $\Phi_{K Z}(A, B)$ in [3], where $\varphi_{K Z}(A, B)=\Phi_{K Z}\left(\frac{1}{2 \pi i} A, \frac{1}{2 \pi i} B\right)$.
} 
It is known that MZV's can be written by iterated integrals as follows.

$$
\begin{array}{r}
\zeta\left(k_{1}, k_{2}, \ldots, k_{m}\right)=\int_{0}^{1} \underbrace{\frac{d u}{u} \circ \cdots \circ \frac{d u}{u} \circ \frac{d u}{1-u}}_{k_{m}} \circ \\
\circ \underbrace{\frac{d u}{u} \circ \cdots \cdots \circ \frac{d u}{1-u}}_{k_{1}}
\end{array}
$$

This expression is due to Kontsevich and Drinfel'd. It can be verified by direct calculations (see, for example, [5] and [8]).

Let

$$
\mathbb{A} \cdot=\underset{w \geqslant 0}{\oplus} \mathbb{A}_{w}=\mathbf{Q}\langle A, B\rangle\left(\subset A_{\mathbb{C}}^{\wedge}\right)
$$

be the non-commutative graded polynomial ring over $\mathbf{Q}$ with two variables $A$ and $B$ with $\operatorname{deg} A=\operatorname{deg} B=1$. Here $\mathbb{A}_{w}$ is the homogeneous degree $w$ part of A. Put

$$
M=A \cdot \mathbb{A} \cdot B=\{A \cdot F \cdot B \mid F \in \mathbb{A} \cdot\}
$$

which is the Q-linear subspace of $\mathbb{A}$. . Define the Q-linear map $Z: M \rightarrow \mathbb{C}$ which is determined by

$$
\begin{gathered}
Z\left(A^{p_{1}} B^{q_{1}} A^{p_{2}} B^{q_{2}} \cdots A^{p_{k}} B^{q_{k}}\right):=\int_{0}^{1} \underbrace{\frac{d u}{u} \circ \cdots \circ \frac{d u}{u}}_{p_{1}} \circ \underbrace{\frac{d u}{1-u} \circ \cdots \circ \frac{d u}{1-u}}_{q_{1}} \\
\circ \frac{d u}{u} \circ \cdots \cdots \cdots \circ \frac{d u}{1-u} \circ \underbrace{\frac{d u}{u} \circ \cdots \circ \frac{d u}{u}}_{p_{k}} \circ \underbrace{\frac{d u}{1-u} \circ \cdots \circ \frac{d u}{1-u}}_{q_{k}} \\
=\zeta(\underbrace{1, \ldots 1}_{q_{k}-1}, p_{k}+1, \underbrace{1, \ldots 1}_{q_{k-1}-1}, p_{k-1}+1, \ldots \ldots, 1, p_{1}+1)
\end{gathered}
$$

for $p_{i}, q_{i} \geqslant 1(1 \leqslant i \leqslant k)$. It is the iterated integral from 0 to 1 obtained by replacing $A$ by $\frac{d u}{u}$ and $B$ by $\frac{d u}{1-u}$.

Definition 3.2.1. The word is an element of $\mathbb{A}$. which is monic and monomial. But exceptionally we shall not call 1 a word. For each word $W$, the weight and depth of $W$ are as follows.

$w t(W):=$ 'the sum of exponents of $A$ and $B$ in $W$ '

$d p(W):=$ 'the sum of exponent of $B$ in $W$ ' 
For example, $A^{3} B A B$ is a word with $w t\left(A^{3} B A B\right)=6$ and $d p\left(A^{3} B A B\right)=$ 2. Note that, by definition, $Z(W) \in Z_{w}^{m}$ (see Definition 1.1.3) for any word $W$ with $w t(W)=w$ and $d p(W)=m$. We can expand uniquely as $\Phi_{K Z}(A, B)=$ $1+\sum_{W \text { :words }} I(W) W$, where each $I(W)$ is a complex number. The following product structure is required to calculate each $I(W)$ explicitly.

Definition 3.2.2. The shuffle product ' $O$ ' is the $\mathbf{Q}$-bilinear map $\circ: \mathbb{A}$. $\times \mathbb{A}$. $\rightarrow \mathbb{A}$., which is defined by

$\underline{\mathbf{S 1}} W \circ 1=1 \circ W=W$

S2 $U W \circ V W^{\prime}=U\left(W \circ V W^{\prime}\right)+V\left(U W \circ W^{\prime}\right) \quad$ where $U, V \in\{A, B\}$,

for all words $W, W^{\prime}$ in $\mathbb{A}$.

For example, $A B \circ A=2 A^{2} B+A B A, A B \circ A B=2 A B A B+4 A^{2} B^{2}$. Note that $Z(W) \cdot Z\left(W^{\prime}\right)=Z\left(W \circ W^{\prime}\right) \quad$ if $W, W^{\prime} \in M$. It is a basic property of iterated integral. There is a natural surjection from $\mathbb{A}$. to $\mathbb{A} \cdot /(B \mathbb{A} \cdot+\mathbb{A} \cdot A)$. By identifying the latter space with $\mathbf{Q} \cdot 1+M(=\mathbf{Q} \cdot 1+A \cdot \mathbb{A} \cdot B)$ we obtain the Q-linear map $f: \mathbb{A} \cdot \hookrightarrow \mathbb{A} \cdot /(B \mathbb{A} \cdot+\mathbb{A} \cdot A) \stackrel{\sim}{\rightarrow} \mathbf{Q} \cdot 1+M \hookrightarrow \mathbb{A}$.

Proposition 3.2.3 (Explicit Formulae). Each coefficient of $\Phi_{K Z}$ $(A, B)=1+\sum_{W: \text { words }} I(W) W$ can be expressed as follows. ${ }^{3}$

(a) When $W$ is in $M, \quad I(W)=(-1)^{d p(W)} Z(W)$.

(b) When $W$ is written as $B^{r} V A^{s}(r, s \geqslant 0, V \in M)$,

$$
I(W)=(-1)^{d p(W)} \sum_{0 \leqslant a \leqslant r, 0 \leqslant b \leqslant s}(-1)^{a+b} Z\left(f\left(B^{a} \circ B^{r-a} V A^{s-b} \circ A^{b}\right)\right) .
$$

(c) When $W$ is written as $B^{r} A^{s}(r, s \geqslant 0)$,

$$
I(W)=(-1)^{d p(W)} \sum_{0 \leqslant a \leqslant r, 0 \leqslant b \leqslant s}(-1)^{a+b} Z\left(f\left(B^{a} \circ B^{r-a} A^{s-b} \circ A^{b}\right)\right) .
$$

Proof. Applying the method (A.15) in [18], we get these expressions.

\footnotetext{
${ }^{3}$ Another explicit formula of the Drinfel'd associator was obtained in [18], but there seems to be an error on the signature in Theorem A.9. Their formula is inconvenient for our present purpose because they expressed each coefficient of MZV in terms of words instead of expressing each coefficient of word in terms of MZV's.
} 
This proposition implies

Property I. Each coefficient of $\Phi_{K Z}(A, B)$ can be written by $M Z V$ 's. More precisely, for each word $W$ with $w t(W)=w$ and $d p(W)=m, I(W)$ is in $Z_{w}^{m}$.

The terms of low degrees of $\Phi_{K Z}(A, B)$ are as follows.

$$
\begin{aligned}
\Phi_{K Z} & (A, B)=1-\zeta(2)[A, B]-\zeta(3)[A,[A, B]]+\zeta(1,2)[[A, B], B] \\
& +\zeta(4)[A,[A,[A, B]]]+\zeta(1,3)[A,[[A, B], B]]-\zeta(1,1,2)[[[A, B], B], B] \\
& +\frac{1}{2} \zeta(2)^{2}[A, B]^{2}+\cdots \cdots
\end{aligned}
$$

\section{$\S 3.3 . \quad$ Relations}

Let $\mathbb{L}_{\mathbb{C}}$ be the completion by degree of the free Lie algebra $\mathbb{L} \cdot \otimes \mathbb{C}$ over $\mathbb{C}$ (for $\mathbb{L}$., see $\S \S 2.2$ ). The Lie algebra $\mathbb{L}_{\mathbb{C}}^{\wedge}$ can be naturally identified with a subspace of $\mathbb{A}_{\mathbb{C}}$ by replacing $[A, B]$ by $A B-B A$. The following property was a key to prove one of the main theorems in [3].

Property II. The Drinfel'd associator satisfies

$$
\left\{\begin{array}{c}
(0) \log \Phi_{K Z}(A, B):=\left\{\sum I(W) W\right\}-\frac{1}{2}\left\{\sum I(W) W\right\}^{2}+\frac{1}{3}\left\{\sum I(W) W\right\}^{3} \\
-\frac{1}{4}\left\{\sum I(W) W\right\}^{4}+\cdots \in\left[\mathbb{L}_{\mathbb{C}}^{\wedge}, \mathbb{L}_{\mathbb{C}}^{\wedge}\right]\left(=\underset{a \geqslant 2}{\oplus}\left(\mathbb{L}_{a} \otimes \mathbf{Q} \mathbb{C}\right)\right) \\
(\mathrm{I}) \Phi_{K Z}(A, B) \Phi_{K Z}(B, A)=1 \\
(\mathrm{II}) e^{\pi i A} \Phi_{K Z}(C, A) e^{\pi i C} \Phi_{K Z}(B, C) e^{\pi i B} \Phi_{K Z}(A, B)=1 \\
\text { for } A+B+C=0 \\
(\mathrm{III}) \Phi_{K Z}\left(x_{1,2}, x_{2,3}\right) \Phi_{K Z}\left(x_{3,4}, x_{4,5}\right) \Phi_{K Z}\left(x_{5,1}, x_{1,2}\right) \\
\Phi_{K Z}\left(x_{2,3}, x_{3,4}\right) \Phi_{K Z}\left(x_{4,5}, x_{5,1}\right)=1 \text { in } \widehat{U \mathfrak{P}^{(5)} \otimes \mathbb{C} .}
\end{array}\right.
$$

Here, $\widehat{U \mathfrak{P}^{(5)}} \otimes \mathbb{C}$ stands for the completion by degree of the universal enveloping algebra of $\mathfrak{P}^{(5)}$ tensored by $\mathbb{C}$. In fact (III) implies (I).

Proposition 3.3.1. Relation (III) implies (I).

Proof. Observe that we have a basic projection of completed noncommutative algebras $p: \widetilde{U \mathfrak{P}^{(5)} \otimes} \mathbb{C} \rightarrow \mathbb{A}_{\mathbb{C}}$ which sends $x_{1,2}, x_{2,3}, x_{3,4}, x_{4,5}$ and 
$x_{5,1}$ to $A, B, A, 0$ and 0 respectively. Since

$$
\begin{gathered}
p\left(\Phi_{K Z}\left(x_{1,2}, x_{2,3}\right) \Phi_{K Z}\left(x_{3,4}, x_{4,5}\right) \Phi_{K Z}\left(x_{5,1}, x_{1,2}\right) \Phi_{K Z}\left(x_{2,3}, x_{3,4}\right) \Phi_{K Z}\right. \\
\left.\times\left(x_{4,5}, x_{5,1}\right)\right)=\Phi_{K Z}(A, B) \Phi_{K Z}(B, A),
\end{gathered}
$$

(III) implies (I), as desired.

Remark 3.3.2. Our above formulae are not exactly the same as those below given in Drinfel'd's original paper [3], however they are equivalent.

- $\log \varphi_{K Z}(A, B) \in\left[\mathbb{L}_{\mathbb{C}}^{\wedge}, \mathbb{L}_{\mathbb{C}}\right]$

- $\varphi_{K Z}(B, A)=\varphi_{K Z}(A, B)^{-1}$

- $e^{\frac{A}{2}} \varphi_{K Z}(C, A) e^{\frac{C}{2}} \varphi_{K Z}(B, C) e^{\frac{B}{2}} \varphi_{K Z}(A, B)=1$

$$
\text { for } A+B+C=0
$$

- $\varphi_{K Z}\left(x_{1,2}, x_{2,3}+x_{2,4}\right) \varphi_{K Z}\left(x_{1,3}+x_{2,3}, x_{3,4}\right)=\varphi_{K Z}\left(x_{2,3}, x_{3,4}\right)$.

$$
\varphi_{K Z}\left(x_{1,2}+x_{1,3}, x_{2,4}+x_{3,4}\right) \varphi_{K Z}\left(x_{1,2}, x_{2,3}\right) \text { in } \widehat{U \mathfrak{P}^{(5)} \otimes \mathbb{C}}
$$

The proof of his formulae relies on some asymptotic behaviors of certain solutions of the KZ equations system on some specified zones. Property I and formulae $(0) \sim($ III) suggest that we can get many algebraic relations among MZV's. In the next section, we shall see that his formulae (equivalently ours) play an essential role to prove our main theorem.

\section{§4. Main Results}

The purpose of this section is to show the close relationship between $N Z$. and $\mathfrak{D}$.

\section{$\S 4.1$ The new-zeta quotient of the Drinfel'd associator}

By Property I ( $\S 3.2)$, the Drinfel'd associator $\Phi_{K Z}(A, B)=1+\sum_{W \text { :words }}$ $I(W) W$ can be regarded as an element of $\underset{w \geqslant 0}{\widehat{\oplus}_{w}}\left(Z_{w} \otimes_{\mathbf{Q}} \mathbb{A}_{w}\right)$.

Definition 4.1.1. The new-zeta quotient of the Drinfel'd associator $\overline{\Phi_{K Z}(A, B)}=\sum_{W \text { :words }} \overline{I(W)} W$ is an element of the Q-linear vector space $\widehat{\oplus}_{w \geqslant 0}$ $\left(N Z_{w} \otimes_{\mathbf{Q}} \mathbb{A}_{w}\right)$ obtained from $\Phi_{K Z}(A, B)$ by replacing each $I(W)$ by $\overline{I(W)}$ which is the image of $I(W)$ by the natural surjection $Z$. $\rightarrow N Z$. $\left(=Z \cdot /\left(\left(Z_{>0}\right)^{2} \oplus Z_{2} \oplus\right.\right.$ $\left.Z_{0}\right)$ ). 
Embed the Lie algebra $\mathbb{L}$. into $\mathbb{A}$. by the map $[A, B] \mapsto A B-B A$ and identify $\mathfrak{D}_{w}$ with

$$
\left\{\begin{array}{l|ll}
f \in \mathbb{A}_{w} & \text { (0) } & f \in[\mathbb{L} ., \mathbb{L} .]\left(=\bigoplus_{k=2}^{\infty} \mathbb{L}_{k}\right) \\
\text { (i) } & f(x, y)+f(y, x)=0 \\
\text { (ii) } & f(x, y)+f(y, z)+f(z, x)=0 \text { for } x+y+z=0 \\
\text { (iii) } & \sum_{i \in \mathbb{Z} / 5} f\left(x_{i, i+1}, x_{i+1, i+2}\right)=0 \text { in } \mathfrak{P}^{(5)}
\end{array}\right\}
$$

by sending $D_{g} \in \mathfrak{D}_{w}$ to $g \in \mathbb{A}_{w}$ for each $w \geqslant 1$. Then we can regard $\mathfrak{D}$. as a subspace of the graded vector space $\mathbb{A}$.

Theorem 4.1.2. The new-zeta quotient of the Drinfel'd associator $\overline{\Phi_{K Z}(A, B)}$ lies on the $\mathbf{Q}$-linear vector space $\underset{w \geqslant 2}{\widehat{\oplus}}\left(N Z_{w} \otimes_{\mathbf{Q}} \mathfrak{D}_{w}\right)$.

Proof.

$$
\begin{aligned}
\Phi_{K Z} & =\exp \log \Phi_{K Z}=1+\log \Phi_{K Z}+\frac{\left(\log \Phi_{K Z}\right)^{2}}{2 !}+\frac{\left(\log \Phi_{K Z}\right)^{3}}{3 !}+\cdots \\
& \equiv 1+\log \Phi_{K Z} \quad \bmod \left(Z_{>0}\right)^{2} .
\end{aligned}
$$

This means that $\overline{\Phi_{K Z}}=\sum_{W \text { :words }} \overline{I(W)} W$ lies on $\underset{w \geqslant 2}{\widehat{\oplus}}\left(N Z_{w} \otimes_{\mathbf{Q}} \mathbb{L}_{w}\right)$. From relations $(0) \sim(\mathrm{III})$ in Property II ( $\S 3.3)$ of the Drinfel'd associator $\Phi_{K Z}(A, B)=$ $1+\sum_{W: \text { words }} I(W) W \in \underset{w \geqslant 0}{\widehat{\oplus}}\left(Z_{w} \otimes_{\mathbf{Q}} \mathbb{A}_{w}\right)$, we find that $\overline{\Phi_{K Z}(A, B)}=\sum_{W \text { :words }}$ $\overline{I(W)} W \in \underset{w \geqslant 2}{\widehat{\oplus}}\left(N Z_{w} \otimes_{\mathbf{Q}} \mathbb{L}_{w}\right)$ satisfies

(i) $\overline{\Phi_{K Z}(A, B)}+\overline{\Phi_{K Z}(B, A)}=0$

(ii) $\overline{\Phi_{K Z}(A, B)}+\overline{\Phi_{K Z}(B, C)}+\overline{\Phi_{K Z}(C, A)}=0$ for $A+B+C=0$

(iii) $\sum_{i \in \mathbb{Z} / 5} \overline{\Phi_{K Z}\left(x_{i, i+1}, x_{i+1, i+2}\right)}=0 \quad$ in $\underset{w \geqslant 1}{\widehat{\oplus}}\left(N Z_{w} \otimes_{\mathbf{Q}} \mathfrak{P}_{w}^{(5)}\right)$

So we find that $\overline{\Phi_{K Z}}$ can be regarded as an element of $\underset{w \geqslant 2}{\widehat{\oplus}}\left(N Z_{w} \otimes \mathbf{Q}_{\mathbf{Q}} \mathfrak{D}_{w}\right)$.

\section{§4.2. Main theorem}

Definition 4.2.1. The graded dual of the stable derivation algebra $\mathfrak{D}^{*}=$ $\underset{w \geqslant 1}{\oplus} \mathfrak{D}_{w}^{*}$ is the graded vector space whose component of degree $w$ is the dual 
vector space of $\mathfrak{D}_{w}$, i.e. $\mathfrak{D}_{w}^{*}=\left(\mathfrak{D}_{w}\right)^{*}$. The depth filtration $\left\{\mathfrak{F}^{m} \mathfrak{D}^{*}\right\}_{m \in \mathbf{N}}$ of $\mathfrak{D}^{*}$. is the ascending filtration:

$$
\{0\}=\mathfrak{F}^{0} \mathfrak{D}^{*} \subseteq \mathfrak{F}^{1} \mathfrak{D}^{*} \subseteq \mathfrak{F}^{2} \mathfrak{D}^{*} \subseteq \mathfrak{F}^{3} \mathfrak{D}^{*} \subseteq \cdots,
$$

where $\mathfrak{F}^{m} \mathfrak{D}_{w}^{*}$ is the vector space of linear forms on $\mathfrak{D}_{w}$ whose restriction to $\mathfrak{F}^{m+1} \mathfrak{D}_{w}$ is zero, i.e. $\mathfrak{F}^{m} \mathfrak{D}_{w}^{*}=\left\{f^{*} \in \mathfrak{D}_{w}^{*}\left|\quad f^{*}\right|_{\mathfrak{F}^{m+1} \mathfrak{D}_{w}} \equiv 0\right\}$.

Then $\left(\mathfrak{D}^{*},\left\{\mathfrak{F}^{m} \mathfrak{D}^{*}\right\}_{m \in \mathbf{N}}\right)$ becomes a filtered graded $\mathbf{Q}$-vector space.

Theorem 4.2.2. There is a canonical surjective Q-linear map of filtered graded vector spaces

$$
\Psi_{D R}:\left(\mathfrak{D}^{*},\left\{\mathfrak{F}^{m} \mathfrak{D}^{*}\right\}_{m \in \mathbf{N}}\right) \rightarrow\left(N Z .,\{N Z . \leqslant m\}_{m \in \mathbf{N}}\right) .
$$

Moreover, it strictly preserves the depth filtration, i.e. $\Psi_{D R}\left(\mathfrak{F}^{m} \mathfrak{D}_{w}^{*}\right)=N Z_{w}^{\leqslant m}$.

Proof. Construction: Decompose $\overline{\Phi_{K Z}}$ as $\overline{\Phi_{K Z}}=\sum_{w=2}^{\infty} \psi_{w}$ where $\psi_{w} \in$ $N Z_{w} \otimes_{\mathbf{Q}} \mathfrak{D}_{w}$ for all $w$. There is the natural isomorphism $N Z_{w} \otimes_{\mathbf{Q}} \mathfrak{D}_{w} \cong$ $H o m_{\mathbf{Q}}\left(\mathfrak{D}_{w}^{*}, N Z_{w}\right)$, thus each $\psi_{w}$ determines a $\mathbf{Q}$-linear map $\Psi_{w}: \mathfrak{D}_{w}^{*} \rightarrow N Z_{w}$. Then we define $\Phi_{D R}$ as follows.

$$
\underset{w=2}{\oplus} \Psi_{w}: \mathfrak{D}^{*}\left(=\underset{w=2}{\oplus} \mathfrak{D}_{w}^{*}\right) \longrightarrow N Z \cdot\left(=\underset{w=2}{\oplus} N Z_{w}\right) .
$$

Surjectivity: Let $w \geqslant 2$. Let $\left\{W^{*} \mid W\right.$ : words, $\left.w t(W)=w\right\}$ be a basis of $\mathbb{A}_{w}^{*}\left(:\right.$ the dual vector space of $\left.\mathbb{A}_{w}\right)$ where

$$
W^{*}\left(W^{\prime}\right)=\left\{\begin{array}{lll}
1 & \text { if } & W=W^{\prime} \\
0 & \text { if } & W \neq W^{\prime}
\end{array}\right.
$$

for all words $W^{\prime} \in \mathbb{A}_{w}$. Denote $\left.W^{*}\right|_{\mathfrak{D}_{w}}$ be the restriction of the map $W^{*}$ on $\mathbb{A}_{w}$ into $\mathfrak{D}_{w}$. Then it is clear that $\left\{\left.W^{*}\right|_{\mathfrak{D}_{w}} \mid W\right.$ : words, $\left.w t(W)=w\right\}$ is a system of generators of the $\mathbf{Q}$-vector space $\mathfrak{D}_{w}^{*}$, i.e.

$$
\left.\mathfrak{D}_{w}^{*}=\left\langle\left. W^{*}\right|_{\mathfrak{D}_{w}}\right| W: \text { words, } w t(W)=w\right\rangle_{\mathbf{Q}} .
$$

Note that $\Psi_{w}\left(\left.W^{*}\right|_{\mathfrak{D}_{w}}\right)=\overline{I(W)}$, thus we find that

$$
\left.\Psi_{w}\left(\mathfrak{D}_{w}^{*}\right)=\langle\overline{I(W)}| W: \text { words, } w t(W)=w\right\rangle_{\mathbf{Q}}
$$


by Proposition 3.2.3

$$
=\langle\overline{Z(W)}| W \in M: \text { words, } w t(W)=w\rangle_{\mathbf{Q}}=N Z_{w} .
$$

This means that the linear map $\Psi_{w}$ is surjective.

Preserving the depth filtration: We see easily that

$$
\left.\mathfrak{F}^{m} \mathfrak{D}_{w}^{*}=\left\langle\left. W^{*}\right|_{\mathfrak{D}_{w}}\right| W: \text { words, } w t(W)=w, d p(W) \leqslant m\right\rangle_{\mathbf{Q}} .
$$

Thus it also follows that

$$
\left.\Psi_{w}\left(\mathfrak{F}^{m} \mathfrak{D}_{w}^{*}\right)=\langle\overline{I(W)}| W: \text { words, } w t(W)=w, d p(W) \leqslant m\right\rangle_{\mathbf{Q}}
$$

by Proposition 3.2.3

$$
\begin{aligned}
& =\langle\overline{Z(W)}| W \in M: \text { words, } w t(W)=w, d p(W) \leqslant m\rangle_{\mathbf{Q}} \\
& =N Z_{w}^{\leqslant m} .
\end{aligned}
$$

This means that the linear map $\Psi_{w}$ strictly preserves the depth filtration.

$\Psi_{D R}$ stands for 'de Rham' and 'Drinfel'd'.

\section{$\S 4.3$. Several corollaries and conjectures}

The surjectivity of $\Psi_{D R}$ implies

Corollary 4.3.1.

(i) $\operatorname{dim}_{\mathbf{Q}} N Z_{w} \leqslant \operatorname{dim}_{\mathbf{Q}} \mathfrak{D}_{w}$.

(ii) More precisely, $\operatorname{dim}_{\mathbf{Q}} N Z_{w}^{\leqslant m} \leqslant \operatorname{dim}_{\mathbf{Q}} \mathfrak{F}^{m} \mathfrak{D}_{w}^{*}=\operatorname{dim}_{\mathbf{Q}}\left(\mathfrak{D}_{w} / \mathfrak{F}^{m+1} \mathfrak{D}_{w}\right)$.

M. Kaneko informed me that Corollary 4.3.1(i) is also appeared in [4], which was deduced from the action of the stable derivation algebra on a certain torsor. Proposition 2.3.1 follows

Corollary 4.3.2.

- $N Z_{w}^{\leqslant m}=N Z_{w}^{\leqslant m+1}$ if $w \equiv m(\bmod 2)$.

- $N Z_{w}^{\leqslant m}=N Z_{w}$ if $\quad m>\frac{w}{2}-1$. 
- $\operatorname{dim}_{\mathbf{Q}} N Z_{w}^{\leqslant 1} \leqslant \begin{cases}1 & w=3,5,7,9, \ldots \\ 0 & w: \text { otherwise. }\end{cases}$

- $\operatorname{dim}_{\mathbf{Q}} N Z_{w}^{\leqslant 2} \leqslant\left\{\begin{array}{cl}0 & w: \text { odd } \\ {\left[\frac{w-2}{6}\right]} & w: \text { even } .\end{array}\right.$

Corollary 4.3.2 (or at least a part of it) seems to have been already found by Don Zagier (cf. [6]).

Example 4.3.3. From the table in Example 2.3.2, we get

\begin{tabular}{|c||c|c|c|c|c|c|c|c|c|c|c|c|}
\hline$w$ & 1 & 2 & 3 & 4 & 5 & 6 & 7 & 8 & 9 & 10 & 11 & 12 \\
\hline $\operatorname{dim}_{\mathbf{Q}} N Z_{w}^{\leqslant 1}$ & 0 & 0 & 1 & 0 & $\leqslant 1$ & 0 & $\leqslant 1$ & 0 & $\leqslant 1$ & 0 & $\leqslant 1$ & 0 \\
\hline $\operatorname{dim}_{\mathbf{Q}} N Z_{w}^{\leqslant 2}$ & 0 & 0 & 1 & 0 & $\leqslant 1$ & 0 & $\leqslant 1$ & $\leqslant 1$ & $\leqslant 1$ & $\leqslant 1$ & $\leqslant 1$ & $\leqslant 1$ \\
\hline $\operatorname{dim}_{\mathbf{Q}} N Z_{w}^{\leqslant 3}$ & 0 & 0 & 1 & 0 & $\leqslant 1$ & 0 & $\leqslant 1$ & $\leqslant 1$ & $\leqslant 1$ & $\leqslant 1$ & $\leqslant 2$ & $\leqslant 1$ \\
\hline $\operatorname{dim}_{\mathbf{Q}} N Z_{w}^{\leqslant 4}$ & 0 & 0 & 1 & 0 & $\leqslant 1$ & 0 & $\leqslant 1$ & $\leqslant 1$ & $\leqslant 1$ & $\leqslant 1$ & $\leqslant 2$ & $\leqslant 2$ \\
\hline \hline $\operatorname{dim}_{\mathbf{Q}} N Z_{w}$ & 0 & 0 & 1 & 0 & $\leqslant 1$ & 0 & $\leqslant 1$ & $\leqslant 1$ & $\leqslant 1$ & $\leqslant 1$ & $\leqslant 2$ & $\leqslant 2$ \\
\hline
\end{tabular}

Example 4.3.4. $\quad$ Using the computation table of the basis of $\mathfrak{D}_{w}$ for $w=11$ and 12 by $\mathrm{H}$. Tsunogai, one can extend the list appearing in [8] to higher weights 11 and 12 as follows.

$$
\begin{aligned}
& Z_{11}=\left\langle\pi^{8} \zeta(3), \pi^{6} \zeta(5), \pi^{4} \zeta(7), \pi^{2} \zeta(3)^{3}, \pi^{2} \zeta(9), \zeta(3)^{2} \zeta(5), \zeta(3) \zeta(3,5),\right. \\
& \underset{d p=1}{\zeta(11), \zeta(2,1,8)}\rangle_{\mathbf{Q}}, \\
& Z_{12}=\left\langle\pi^{12}, \pi^{6} \zeta(3)^{2}, \pi^{4} \zeta(3) \zeta(5), \pi^{4} \zeta(3,5), \pi^{2} \zeta(3) \zeta(7), \pi^{2} \zeta(5)^{2}, \pi^{2} \zeta(3,7),\right. \\
& \left.\zeta(3)^{4}, \zeta(3) \zeta(9), \zeta(5) \zeta(7), \underset{d p=2}{\zeta(3,9), \zeta(p=4} \underset{d p}{(2,1,1,8)}\right\rangle_{\mathbf{Q}} .
\end{aligned}
$$

Let $\left\{d_{k}^{\prime}\right\}_{k=0}^{\infty}$ be the sequence determined by the following series.

$$
\sum_{k=0}^{\infty} d_{k}^{\prime} t^{k}:=\frac{1}{1-t^{2}} \prod_{w=1}^{\infty} \frac{1}{\left(1-t^{w}\right)^{\operatorname{dim}_{\mathbf{Q}} \mathfrak{D}_{w}}} .
$$

Then $d_{k}^{\prime}$ gives the dimension of the degree $k$-part of the graded polynomial algebra infinitely generated by $z_{i, j}\left(1 \leqslant i, 1 \leqslant j \leqslant \operatorname{dim}_{\mathbf{Q}} \mathfrak{D}_{i}\right)$ with $\operatorname{deg} z_{i, j}=i$ and $\pi^{2}$ with $\operatorname{deg} \pi^{2}=2$. Thus we get the following dimension bounding of the MZV algebra. 
Corollary 4.3.5.

$$
\operatorname{dim}_{\mathbf{Q}} Z_{w} \leqslant d_{w}^{\prime} \quad \text { for all } w .
$$

Here, the equality holds if and only if $\Psi_{D R}: \mathfrak{D}^{*} . \rightarrow N Z$. is an isomorphism and $Z$. is polynomial algebra.

Proof. It follows immediately from

$$
\begin{aligned}
\sum_{k=0}^{\infty} \operatorname{dim}_{\mathbf{Q}} Z_{k} \cdot t^{k} & \leqslant \frac{1}{1-t^{2}} \prod_{w=1}^{\infty} \frac{1}{\left(1-t^{w}\right)^{\operatorname{dim}_{\mathbf{Q}} N Z_{w}}} \\
& \leqslant \frac{1}{1-t^{2}} \prod_{w=1}^{\infty} \frac{1}{\left(1-t^{w}\right)^{\operatorname{dim}_{\mathbf{Q}} \mathfrak{D}_{w}}}=\sum_{k=0}^{\infty} d_{k}^{\prime} t^{k}
\end{aligned}
$$

by Corollary 4.3.1(i). Here, for two formal power series $P(t), Q(t)$ in $\mathbf{Q}[[t]]$, $P(t) \geqslant Q(t)$ means that the formal power series $P(t)-Q(t)$ has all of its coefficients non-negative.

From the table in Example 2.3.2, we see that $d_{w}^{\prime}=d_{w}$ for $w \leqslant 12$. Here, $d_{w}$ is the conjectured dimension of $Z_{w}$ in $\S \S 1.2$. Moreover, we find that

Lemma 4.3.6. $\quad$ Suppose that Conjecture 2.4.1(3) for $\mathfrak{D}$. is true. Then

$$
d_{w}^{\prime} \leqslant d_{w} \quad \text { for all } w .
$$

The equality holds if and only if Conjecture 2.4.1(2) for $\mathfrak{D}$. holds.

Proof. Let $\mathbb{F} .=\underset{m \geqslant 1}{\oplus} \mathbb{F}_{m}$ be the free graded Lie algebra over $\mathbf{Q}$ generated by $e_{m}$ with $\operatorname{deg} e_{m}=m(m=3,5,7, \ldots)$. From the assumption of this lemma, $\operatorname{dim}_{\mathbf{Q}} \mathfrak{D}_{w} \leqslant \operatorname{dim}_{\mathbf{Q}} \mathbb{F}_{w}$ for all $w$. So we get

$$
\begin{aligned}
\sum_{k=0}^{\infty} d_{k}^{\prime} \cdot t^{k} & =\frac{1}{1-t^{2}} \prod_{w=1}^{\infty} \frac{1}{\left(1-t^{w}\right)^{\operatorname{dim}_{\mathbf{Q}} \mathfrak{D}_{w}}} \\
& \leqslant \frac{1}{1-t^{2}} \prod_{w=1}^{\infty} \frac{1}{\left(1-t^{w}\right)^{\operatorname{dim}_{\mathbf{Q}} \mathbb{F}_{w}}}=\sum_{k=0}^{\infty} d_{k} \cdot t^{k}
\end{aligned}
$$

A short proof of the last equality was given in [8]. The equality $d_{k}^{\prime}=d_{k}$ for all $k$ holds if and only if $\operatorname{dim}_{\mathbf{Q}} \mathfrak{D}_{w}=\operatorname{dim}_{\mathbf{Q}} \mathbb{F}_{w}$ for all $w$, which is equivalent to saying that $\mathfrak{D}$. is the free graded Lie algebra generated by one element in each degree $m(=3,5,7, \ldots)$. 
We can deduce the following proposition from Corollary 4.3.5.

Proposition 4.3.7. Suppose that Conjecture 2.4.1(3) for $\mathfrak{D}$. is true, i.e. $\mathfrak{D}$. is generated by one element in each degree $m(=3,5,7,9, \ldots)$. Then

$$
\operatorname{dim}_{\mathbf{Q}} Z_{w} \leqslant d_{w} \quad \text { for all } w
$$

Namely, the conjecture on the structure of the stable derivation algebra which arose from the study of the Galois representation on $\pi_{1}\left(\mathbf{P}_{\overline{\mathbf{Q}}}^{1}-\{0,1, \infty\}\right)$ implies the upper-bounding part of the Dimension Conjecture of multiple zeta values $(\S \S 1.2)$ !

From Corollary 4.3.5, the validity of the Dimension Conjecture and the Conjecture 2.4.1(2),(3) for $\mathfrak{D}$. would imply that $Z$. might be a polynomial algebra $([8])$ and the following

Conjecture 4.3.8. The surjective linear map

$$
\Psi_{D R}:\left(\mathfrak{D}^{*},\left\{\mathfrak{F}^{m} \mathfrak{D}^{*}\right\}_{m \in \mathbf{N}}\right) \rightarrow\left(N Z .,\left\{N Z:{ }^{m}\right\}_{m \in \mathbf{N}}\right)
$$

is an isomorphism as filtered graded $\mathbf{Q}$-vector space.

But it may be hard to prove the injectivity, since it implies deep results of transcendental number theory.

Remark 4.3.9. Particularly Conjecture 4.3.8 implies that the dual vector space $N Z^{*}$. might form a sub-Lie algebra of $\mathfrak{D}$. by the Lie bracket $\delta$ of $\mathfrak{D}$.. This indicates that, for example, the map $N Z_{3}^{*} \otimes N Z_{5}^{*} \rightarrow N Z_{8}^{*}$ induced from the Lie bracket $\delta: \mathfrak{D}_{3} \otimes \mathfrak{D}_{5} \rightarrow \mathfrak{D}_{8}$ might be an injection, which means especially $(0 \leqslant) \operatorname{dim}_{\mathbf{Q}} N Z_{5} \leqslant \operatorname{dim}_{\mathbf{Q}} N Z_{8}(\leqslant 1)$ because $\operatorname{dim}_{\mathbf{Q}} N Z_{3}=1$. However showing this inequality looks a difficult problem (at least for the author) related to transcendental number theory.

On the algebraic relations among MZV's, the author raises the following conjecture.

Conjecture 4.3.10. All of the algebraic relations among the MZV's can be deduced from the relations of the Drinfel'd associator $(0) \sim(I I I)$ in Property II (§§3.3). 
Suppose that Conjecture 4.3.10 holds. Then Conjecture 4.3.8 and Direct Sum Conjecture ( $\$ 1.2)$ also hold. By the way, is it possible to deduce all of the algebraic relations among the MZV's which were found till now from $(0) \sim(\mathrm{III})$ ? This question does not seem so trivial at all.

Remark 4.3.11 ([2]§§18.13-18.17). P. Deligne deduced the Euler's formula $\zeta(2 n)=\frac{-(2 \pi i)^{2 n}}{2(2 n) !} B_{n}\left(n \in \mathbf{N}, B_{n}\right.$ : the Bernoulli number $)$ from $(0) \sim(\mathrm{II})$. Since his proof is interesting, we give its brief sketch.

By (I) and (II), we get

$$
\begin{aligned}
& e^{\pi i C} \Phi_{K Z}(C, B)^{-1} e^{\pi i B} \Phi_{K Z}(A, B) e^{\pi i A} \\
& \quad=e^{-\pi i C} \Phi_{K Z}(C, B)^{-1} e^{-\pi i B} \Phi_{K Z}(A, B) e^{-\pi i A}
\end{aligned}
$$

He calculated the image of both hand sides of the above equality modulo $\exp \mathfrak{F}^{2} \mathbb{L}_{\mathbb{C}}^{\wedge}$ in $[2] \S 18.16$ as follows:

$$
\begin{aligned}
&(\mathrm{LHS}) \equiv \exp \left[\frac{e^{-\pi i(a d A)}-1+e^{-\pi i(a d A)}\left\{\pi i(a d A)+2 \sum_{n=1}^{\infty} \zeta(2 n)(a d A)^{2 n}\right\}}{a d A}(B)\right] \\
& \bmod \exp \mathfrak{F}^{2} \mathbb{L}_{\widehat{C}}^{\wedge}, \\
&(\mathrm{RHS}) \equiv \exp \left[\frac{e^{\pi i(a d A)}-1+e^{\pi i(a d A)}\left\{-\pi i(a d A)+2 \sum_{n=1}^{\infty} \zeta(2 n)(a d A)^{2 n}\right\}}{a d A}(B)\right] \\
& \bmod \exp \mathfrak{F}^{2} \mathbb{L}_{\overparen{C}}^{\wedge},
\end{aligned}
$$

from which he obtained

$$
\sum_{n=1}^{\infty} \zeta(2 n)(a d A)^{2 n}(B)=\sum_{n=1}^{\infty} \frac{-(2 \pi i)^{2 n}}{2(2 n) !} B_{n}(a d A)^{2 n}(B) .
$$

\section{§5. Some Comparisons between 'Galois Side' and 'Hodge Side'}

For each $m \in \mathbf{N}$, the element $D=D_{f} \in \mathfrak{D}_{m}$ determines the unique rational number $c_{m}(D)$ by the congruence $f \equiv c_{m}(D) \cdot(a d x)^{m-1}(y) \bmod \mathfrak{F}^{2} \mathbb{L}_{m}$. Y. Ihara constructed a canonical Q-linear map $c_{m}: \mathfrak{D}_{m} \rightarrow \mathbf{Q}$ in [16] which is defined by $D \mapsto c_{m}(D)$. It is shown in [16] that $c_{m} \in \mathfrak{D}_{m}^{*}$ is non-vanishing if and only if $m$ is odd and $m \geqslant 3$. 
For $\sigma \in \operatorname{Gal}(\overline{\mathbf{Q}} / \mathbf{Q})$, let $\tilde{\kappa}_{m}^{(l)}(\sigma)$ be the unique $l$-adic integer satisfying

$$
\begin{gathered}
\prod_{a \in\left(\mathbf{Z} / l^{n} \mathbf{Z}\right) \times}\left(\left[\left\{\left(\zeta_{l^{n}}^{a}-1\right)^{\left\langle a^{m-1}\right\rangle}\right\}^{\frac{1}{l^{n}}}\right]^{\sigma} /\left\{\left(\zeta_{l^{n}}^{\chi(\sigma) a}-1\right)^{\left\langle a^{m-1}\right\rangle}\right\}^{\frac{1}{l^{n}}}\right) \\
=\zeta_{l^{n}}^{\left(l^{m-1}-1\right) \cdot \tilde{\kappa}_{m}^{(l)}(\sigma)}
\end{gathered}
$$

for all $n \in \mathbf{N}$. Here $\zeta_{l^{n}}=\exp \left(\frac{2 \pi i}{l^{n}}\right),\left\langle a^{m-1}\right\rangle$ is the representative of $a^{m-1} \bmod l^{n}$ with $0<\left\langle a^{m-1}\right\rangle<l^{n}$ and $\chi$ is the $l$-adic cyclotomic character. It is nonvanishing if and only if $m$ is odd and $m \geqslant 3$ ([19]). The map $\tilde{\kappa}_{m}^{(l)}(m \geqslant$ 3 , odd) is called the $m$-th Soule element. It represents a non-trivial generator of $H_{\text {ét }}^{1}\left(\operatorname{Spec} \mathbf{Q}, \mathbf{Q}_{l}(m)\right)$, which is of rank 1 for odd $m \geqslant 1$ and rank 0 for other $m(\geqslant 1)$.

Taking the dual of the embedding $\Psi_{l}: \mathfrak{g}^{l} \cdot \hookrightarrow \mathfrak{D} \cdot \otimes_{\mathbf{Q}} \mathbf{Q}_{l}$ which is associated with the Galois representation on the pro- $l$ fundamental group $\pi_{1}^{l}\left(\mathbf{P}_{\overline{\mathbf{Q}}}^{1}-\right.$ $\{0,1, \infty\}$ ) (see $\S \S 2.4$ ), we get a surjective linear map $\Psi_{l}^{*}: \mathfrak{D}^{*} \otimes_{\mathbf{Q}} \mathbf{Q}_{l} \rightarrow \mathfrak{g}^{l *}$. It is shown in [16] that the image of $c_{m} \otimes_{\mathbf{Q}} 1$ by the map $\Psi_{l}^{*}: \mathfrak{D}^{*} \otimes_{\mathbf{Q}} \mathbf{Q}_{l} \rightarrow \mathfrak{g}^{l *}$ is $\frac{1}{(m-1) !} \kappa_{m}^{(l)}$, where $\kappa_{m}^{(l)}: \mathfrak{g}_{m}^{l} \rightarrow \mathbf{Q}_{l}$ is the induced $\mathbf{Q}_{l}$-linear map by $\tilde{\kappa}_{m}^{(l)}$. On the other hand, we find that the image of $c_{m}$ by $\Psi_{D R}: \mathfrak{D}^{*} \rightarrow N Z$. is $\overline{-\zeta(m)}$ by Proposition 3.2.3(a). We can make a comparison between 'the de Rham world' and 'the $l$-adic world' as follows.

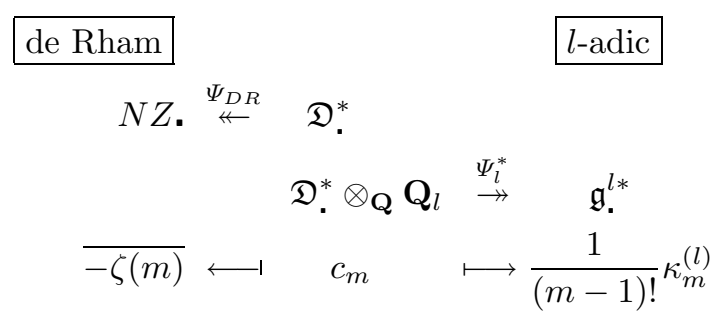

Conjecture 4.3.8 and Conjecture 2.4.1 expect that both $\Psi_{D R}$ and $\Psi_{l}^{*}$ are isomorphisms.

\section{References}

[1] Belyı̆, G. V., Galois extensions of a maximal cyclotomic field, (Russian) Izv. Akad. Nauk SSSR Ser. Mat., 43 (1979), 267-276, 479.

[2] Deligne, P., Le groupe fondamental de la droite projective moins trois points, Galois groups over $Q$ (Berkeley, CA, 1987), 79-297, Math. Sci. Res. Inst. Publ., 16, Springer, New York-Berlin, 1989. 
[3] Drinfel'd, V. G., On quasitriangular quasi-Hopf algebras and a group closely connected with $\operatorname{Gal}(\bar{Q} / Q)$, Leningrad Math. J., 2 (1991), 829-860.

[4] Etingof, P. and Schiffmann, O., Lectures on quantum groups, Cambridge, International Press, 1998.

[5] Goncharov, A. B., The double logarithm and Manin's complex for modular curves, Math. Res. Lett., 4 (1997), 617-636.

[6] , Multiple polylogarithms, cyclotomy and modular complexes, Math. Res. Lett., 5 (1998), 497-516

[7] $ـ$, The dihedral Lie algebras and Galois symmetries of $\pi_{1}^{l}\left(P^{1}-\left\{0, \infty, \mu_{N}\right\}\right)$, math.AG/0009121, preprint in 2000.

[8] _ Multiple zeta-values, Galois groups, and geometry of modular varieties, math.AG/0005069, preprint in 2000.

[9] Grothendieck, A., Esquisse d'un programm, Geometric Galois actions, 1, 5-48, Edited by Leila Schneps and Pierre Lochak. London Math. Soc. Lecture Note Ser., 242, Cambridge University Press, Cambridge, 1997.

[10] Hain, R. and Matsumoto, M., Weighted Completion of Galois Groups and Some Conjectures of Deligne, math/AG0006158, preprint in 2000.

[11] Ihara, Y., The Galois representation arising from $P^{1}-\{0,1, \infty\}$ and Tate twists of even degree, Galois groups over $Q$ (Berkeley, CA, 1987), 299-313, Math. Sci. Res. Inst. Publ., 16, Springer, New York-Berlin, 1989.

[12] _ Braids, Galois groups, and some arithmetic functions, Proceedings of the International Congress of Mathematicians, Vol. I, II (Kyoto, 1990), 99-120, Math. Soc. Japan, Tokyo, 1991.

[13] _ Automorphisms of pure sphere braid groups and Galois representations, The Grothendieck Festschrift, Vol. II, 353-373, Progr. Math., 87, Birkhäuser Boston, Boston, MA, 1990.

[14] Math., 80 (1992), 135-153.

[15] On the embedding of $\operatorname{Gal}(\bar{Q} / Q)$ into $\widehat{\mathrm{GT}}$, London Math. Soc. Lecture Note Ser., 200, The Grothendieck theory of dessins d'enfants (Luminy, 1993), 289-321, Cambridge Univ, Press, Cambridge, 1994.

[16] Some arithmetic aspects of Galois actions on the pro- $p$ fundamental group of $\mathbb{P}^{1}-\{0,1, \infty\}$, RIMS-1229, preprint in 1999.

[17] Kassel, C., Quantum groups, Graduate Texts in Mathematics, 155. Springer-Verlag, New York, 1995.

[18] Le, T.T.Q. and Murakami, J., Kontsevich's integral for the Kauffman polynomial, Nagoya Math. J., 142 (1996), 39-65.

[19] Soulé, C., On higher p-adic regulators, Algebraic K-theory, Evanston 1980 (Proc. Conf., Northwestern Univ., Evanston, III., 1980), pp. 372-401, Lecture Notes in Math., 854, Springer, Berlin-New York, 1981.

[20] Rivoal, T., La fonction Zeta de Riemann prend une infinite de valeurs irrationnelles aux entiers impairs, Comptes rendus de l'Academie des sciences de Paris, ser 1, pp. 267-270, 2000

[21] Terasoma, T., Mixed Tate Motives and Multiple Zeta Values, math.AG/0104231, preprint in 2001.

[22] Tsunogai, H., On ranks of the stable derivation algebra and Deligne's problem, Proc. Japan Acad. Ser. A Math. Sci., 73 (1997), 29-31.

[23] Zagier, D., Values of zeta functions and their applications, First European Congress of Mathematics, Vol. II (Paris, 1992), 497-512, Progr. Math., 120, Birkhäuser, Basel, 1994. 